\title{
Visually perceived motion in depth resulting from proximal changes. II
}

\author{
HANS MARMOLIN* \\ Lniversity of Uppsala, Svartbäcksgatan 10. S.75320 Uppsala, Sweden
}

\begin{abstract}
According to a model for motion and form perception proposed by Johansson (1964), every two-dimensional change in a changing proximal stimulation is projected out as a motion in depth. The model assumes that the amount of perceived relative motion (the fraction between the perceived amount of motion of the object and the perceived initial distance to the object) is determined only by the amount of relative change (the fraction between the absolute amount of change and the initial size). The aim of the present study was to test this hypothesis by studying the effect of some other variables on perceived relative motion in depth. As stimuli. continuously shrinking and growing squares were used. No effects were found when varying the absolute amount of change. Neither did the rate of change influence the perceived relative motion in any important way. The only variable that gave rise to strong and systematic effects on perceived relative motion was the initial distance to the perceived object. The greater the initial distance, the less relative motion was perceived.
\end{abstract}

In a previous article (Marmolin, 1973), a series of experiments was reported concerning the relation between perceived relative distance of motion in depth and relative amount of proximal change. The aim of these experiments was to test the validity of a formula for the stimulus-percept relationship derived from a model proposed by Johansson (1964). According to the model. all change in a regular transformation pattern (a pattern with equal change in the $\mathrm{X}$ and $\mathrm{Y}$ dimensions) is to be projected out as a motion in depth. The equation for the stimulus-percept relation can then be written (notations, see Fig. 1, Marmolin, 1973)

$$
\mathrm{Z} / \mathrm{K}=\left(\frac{\Delta \mathrm{x}}{\mathrm{X}-\Delta \mathrm{x}}\right)^{\mathrm{n}}
$$

The earlier study (Marmolin. 1973) showed that Eq. 1 does not predict the perceived amount of relative motion in depth under the condition used. However, an alternative equation, based on the assumption that a fixed proportion of change (a) is not projected out as a motion, agreed quite well with the data. This equation was written

$$
\mathrm{Z} / \mathrm{K}=\frac{\Delta \mathrm{x}-\mathrm{a} \cdot \Delta \mathrm{x}}{\mathrm{X}-(\Delta \mathrm{x}-\mathrm{a} \cdot \Delta \mathrm{x})}
$$

The obtained deviations from the model can be explained in many different ways. In order to decide between alternative explanations, it is necessary to know if there are other variables, besides amount of relative change, that influence the perceived relative motion in depth. The model assumes that the proximal change is

*The author is indebted to Professor Gunnar Johansson for valuable discussions on this project and for his constructive criticism of the manuscript. This investigation was made possible by grants to Professor Johansson from the Swedish Council for Science Research and the Tricentennial liund of the Bank of Sweden. the only important variable, but this assumption has not yet been systematically tested.

The aim of the present study was to study the effects of rate of change, perceived initial distance to the perceived object, and absolute amount of change on perceived relative motion in depth.

\section{THE EFFECT OF RATE OF CHANGE}

\section{Experiment 1}

In the earlier experiments (Marmolin, 1973; Marmolin \& Ulfberg, 1972: Johansson, 1964), the transformation patterns shrank or grew periodically with a constant rate of change, that is $\Delta \mathrm{x}=\mathrm{r} \cdot \mathrm{t}(\mathrm{t}=\mathrm{time}, \mathrm{r}$ is a constant $)$.

The aim of Experiment 1 was to study the effect of rate of change on perceived motion in depth. In accordance with Eq. 1, the rate of change will be expressed as relative rate of change (percent change/second). In addition, the initial size of the pattern and the relative amount of change were varied.

The effects of these variables on perceived motion were studied indirectly, rather than directly, by means of a matching paradigm. The S's task was to adjust the amount of change in a variable pattern with a certain rate of change (and initial size) so that the motion of this pattern matched the motion of a standard pattern with a different rate of change (and/or initial size). By this matching paradigm, a threshold PSE-Z (point of subjective equality of motion in depth) was obtained. This threshold corresponds to the relative amount of change in the variable pattern necessary to produce equal amount of relative motion in depth as the standard pattern. If, for example, there is any effect of rate of change, then standards of different rate of change ought to give rise to different values of PSE-Z.

\section{Method}

Experimental technique. The standard and variable pattern 


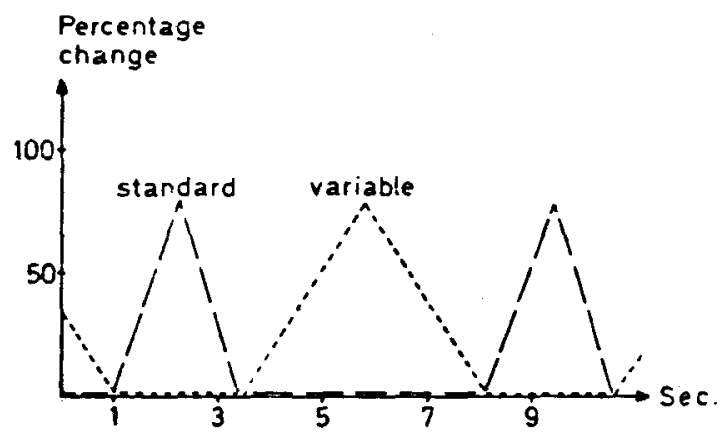

Fig. 1. The change of the contour of the variable pattern and of one of the standards in Experiment 1.

were filled squares of homogeneous brightness that were continuously shrinking and growing (identical changes in the $\mathrm{X}$ and $Y$ dimensions) with a constant rate of change. The two patterns were presented simultaneously but with successively. changing size. i.e.. the $S s$ perceived two squares. one moving in depth while the other was standing still (Fig. 1).

The brightness of the squares changed with the changes in size. This. however. is a rather weak source of information about depth. and the effect of brightness ought to be the same on the standard and the variable patterns. The brightness of the standards of different initial size was adjusted to approximately the same magnitude.

The transformation patterns were generated by means of two high-frequency generators and an analog computer connected to a double-beam oscilloscope. Essentially the same technique had been used earlier by Johansson (1964), but in the experiments to be reported here. the size-modulating wave was obtained by integrating a constant voltage. Amount of change was determined by time of integration and rate of change by the integrated voltages (see Fig. 1), but these values have been transformed to percent change and percent change second.

In order to avoid the perception of a screen carrving the transformation patterns, the $S$ viewed them through a collimating lens system, as in the earlier study (Aarmolin. 1973). The room was darkened, and the two squares were presented beside each other (the centers at the same horizontal axis), with a distance between the contours of about $4 \mathrm{deg}$ of visual angle.

Subjects and procedure. Eight standards were used, consisting of all possible combinations between two relative rates of change $116.5 \% \mathrm{sec}$ and $66 \mathrm{c} / \mathrm{sec})$, two initial sizes of the contours $(5.5$ and $2.8 \mathrm{deg})$. and two relative amounts of change (40\% and $80 \%)$ (see Table 1). The variable pattern had an initial size of $5.5 \mathrm{deg}$ and a relative rate of change of $33 \% / \mathrm{sec}$.

The threshold PSE-Z was measured by a variant of the method of limits, the up-and-down method. This method had earlier been used in similar experiments (Marmolin \& Ulfberg. 1972) and was then found to give good estimates of threshold. In the up-and-down method, the threshold PSE-Z is continuously measured by increasing the amount of change in the variable pattern as the Ss judge this motion to be smaller than that of the standard, and vice versa. The amount of change in the variable pattern was varied by the $E$ in steps of $3.3 \%$ by varying the time of change.

The standard and variable pattern were presented simultaneously and moved successively, as shown in Fig. 1. and at each presentation of a certain variable pattern, the motions of the patterns were shown as many times as necessary for the $\mathrm{Ss}$ to judge the motion of the variable pattern as being smaller or larger than that of the standard.

The different standard patterns were presented once in randomized order, and at each presentation of a standard pattern. PSE-Z was measured five times. Six students of psychology participated as Ss.

\section{Results and Conclusions}

The result of Experiment 1 is summarized in Table 1. As this table shows, there are almost no differences in PSE. $Z$ (means over $S$ ) for standards of the same relative amount of change.

An analysis of variance (mixed model: $S$ by Size by Relative Rate of Change by Relative Amount of Change) revealed that none of the experimental variables had significant effects $(p<.05)$, except, of course, for relative amount of change. Nor were the interactions between these variables significant. The effect of $S$ and interaction with $S$ was not interpreted, as no "true" replications were made. There was, however, no evidence in data of different systematic effects for different Ss.

Experiment 1, then, shows that during the condition used neither initial size of the contour nor relative rate of change influences perceived motion in depth. It should be noticed that for patterns with the same relative rate of change, the absolute rate of change varied with the initial size of the pattern (the absolute rate of change $=$ the relative rate of change $X$ the initial size of the pattern). As no effect of relative rate of change and initial size was found, it can be concluded that the absolute rate of change does not influence perceived motion in depth either.

\section{Experiment 2}

In Experiment 1. a constant rate of change was used. The distal velocity function producing a constant rate of change can be expressed as (according to Eq. 1, $n=1$ )

$$
V_{d}=\frac{K \cdot R}{(1-R \cdot t)^{2}}
$$

where $V_{d}=$ the distal velocity: $K$, the initial distance; $t$, time; and $R$, the proximal relative rate of change.

In the earlier experiments (Marmolin, 1973), the majority of the Ss underestimated the motion. In these experiments, very large changes were used (up to $98.5 \%$ ). If the Ss had functioned according to Eq. 1, the perceived distal velocity ought to have changed according to Eq. 3 in a very dramatic and "unrealistic"

Table 1

PSE-Z for the Different Standard Patterns of Experiment 1 *

\begin{tabular}{|c|c|c|c|c|c|}
\hline \multirow{3}{*}{$\begin{array}{c}\text { Relative } \\
\text { Rate of } \\
\text { Change } \\
\text { in } \\
\text { Percent/Sec }\end{array}$} & & \multirow{2}{*}{\multicolumn{2}{|c|}{$\begin{array}{l}40 \% \text { Change } \\
\text { Maximal Size }\end{array}$}} & \multirow{2}{*}{\multicolumn{2}{|c|}{$\begin{array}{l}80 \% \text { Change } \\
\text { Maximal Size }\end{array}$}} \\
\hline & & & & & \\
\hline & & 5.5 Deg & $2.8 \mathrm{Deg}$ & $5.5 \mathrm{Deg}$ & 2.8 Deg \\
\hline 16.5 & $\begin{array}{l}\text { Mean } \\
\text { SD }\end{array}$ & $\begin{array}{r}42.5 \\
5.5\end{array}$ & $\begin{array}{r}42.5 \\
4.5\end{array}$ & $\begin{array}{r}82.5 \\
3.3\end{array}$ & $\begin{array}{r}82.5 \\
3.0\end{array}$ \\
\hline 66.0 & $\begin{array}{l}\text { Mean } \\
\text { SD }\end{array}$ & $\begin{array}{r}39.5 \\
4.3\end{array}$ & $\begin{array}{r}42.5 \\
7.5\end{array}$ & $\begin{array}{r}82.5 \\
6.5\end{array}$ & $\begin{array}{r}79.5 \\
5.3\end{array}$ \\
\hline
\end{tabular}

*The means and SDs of the individual means are given: PSE-Z is expressed in percent change. 
way. It can be argued that the simple decoding principles proposed in the model are not applicable in such "unrealistic" situations. Thus, with a more realistic rate of change-function, the underestimations would disappear and Eq. 1 would be valid.

In accordance with this line of reasoning, the problem of Experiment 2 was set forth as follows: Dose the type of rate of change function influence perceived motion in depth? The rate of change functions studied were: (1) The a pattern-A pattern with a retarded rate of change function geometrically corresponding to a constant distal velocity and therefore assumed to be a rather "realistic" one. This rate of change function can be expressed as $\Delta x / x=b \cdot t /(1+b \cdot t)$, where $t=$ time, $\mathrm{b}$ is a constant, and $\mathrm{K}$ in Eq. 3 is assumed to be 1 . (2) The $b$ pattern-A pattern with a constant rate of change, as in Experiment 1, that is, $\Delta x / x=b \cdot t$. (3) The $c$ pattern-A pattern with an accelerated rate of change, $\Delta \mathrm{x} / \mathrm{x}=\mathrm{b} \cdot \mathrm{t}^{2}$. This pattern was chosen as a still more "unrealistic" pattern than the b pattern.

It was predicted, in accordance with the discussion above, that the a pattern would produce a greater amount of motion than the $b$ and $c$ patterns.

\section{Method}

Five students of psychology participated as Ss. The same experimental method and technique of generating and presenting the transformation patterns used in Experiment 1 were used. The different rate of change functions were generated by the analog computer according to the equations above (see Fig. 3). As a variable pattern. Pattern b was chosen with a rate of change of $21 \% / \mathrm{sec}$, and as standards, Patterns a and $\mathrm{c}$, with $80 \%$ change and with two mean rates of change, $21 \% / \mathrm{sec}$ and $42 \% / \mathrm{sec}$. The initial size of the contour of the patterns was $5.5 \mathrm{deg}$ of visual angle.

The same matching paradigm and method of measuring PSE-Z was used as in Experiment 1. The standards were presented twice. once with the change of the variable pattern smaller than the expected threshold, once with larger change. At each presentation of the standards, four measures of PSE- $Z$ were obtained.

\section{Results and Conclusions}

The results are summarized in Table 2 . An analysis of variance (mixed model, Subject by Mean Rate of Change by Rate of Change Function) showed that the effect of

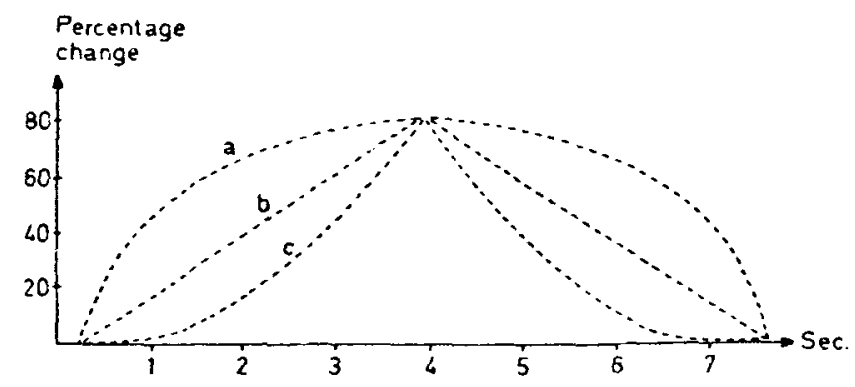

Fig. 2. The rate of change functions of the $a, b$, and c patterns in Experiment 2.
Table 2

PSE-Z (in Percent Change) for the Different Standard Patterns of Experiment 2*

\begin{tabular}{cccc}
\hline \multirow{2}{*}{$\begin{array}{c}\text { Mean Rate } \\
\text { of Change } \\
\text { in Percent/Sec }\end{array}$} & & \multicolumn{2}{c}{ Rate of Change Function } \\
\cline { 3 - 4 } 21 & Mean & 81 & a Pattern \\
\hline \multirow{2}{*}{42} & SD & 1.6 & 76 \\
& Mean & 82 & 3.9 \\
& SD & 1.9 & 78 \\
\hline
\end{tabular}

*The means and SDs of the individual means are given.

rate of change function was significant $(p<.05)$, but not the mean rate of change or the interaction between these variables. As in Experiment 1, the effect of $S$ and interactions with $S$ were not interpreted, but an inspection of individual data showed that all Ss conformed to the general trend. However, there was some variation in the magnitude of the effect.

As predicted, the "realistic" a pattern produced a greater amount of perceived motion in depth than the more "unrealistic" c pattern. However, the effect was very weak. According to the result of the earlier study (Marmolin, 1973), the difference in PSE-Z between the $a$ and $c$ patterns can be estimated to correspond to an increase in perceived motion of about $10 \%$. This must be regarded as a very weak effect in comparison with the variation in rate of change. For example, at $80 \%$ change (42\%/sec mean rate of change), the rate of change of the a pattern was $8 \% / \mathrm{sec}$ and of the c pattern, $80 \% / \mathrm{sec}$. Thus, explanations of the deviations obtained in the earlier study (Marmolin, 1973) from Eq. 1 can hardly be based on the variable rate of change.

From this experiment, one cannot conclude that changes in rate of change form an effective variable contrary to rate of change, even if this seems plausible. In Experiment 1, no effect of rate of change for patterns with constant rate of change was found, but the variation in this experiment was from $16.5 \% / \mathrm{sec}$ to $68 \% / \mathrm{sec}$, and it is possible that a greater variation would have given the same effect as in Experiment 2.

Experiment 2, then, suggests that rate of change or at least changes in rate of change may have some effect, but not a very large one, on perceived motion in depth.

\section{EFFECTS OF PERCEIVED INITIAL DISTANCE AND ABSOLUTE AMOUNT OF CHANGE}

\section{Experiment 3}

In the earlier experiments (Marmolin, 1973), large interindividual differences in amount of perceived relative motion were found $A s$ in these experiments, the Ss had to be isolated from all sources of information about depth other than the proximal change; there was no information available about the initial distance. Therefore, different Ss placed the object at different distances. (Thirteen Ss, who were asked to 
Table 3

Percent Change (Mean) Producing a Motion Cortesponding to the Initial Distance of the Different Patterns of Experiment 3

\begin{tabular}{|c|c|c|c|c|c|}
\hline \multirow[b]{2}{*}{ Si } & \multirow{2}{*}{$\begin{array}{c}\text { Experi- } \\
\text { ment }\end{array}$} & \multicolumn{4}{|c|}{ Maximal Size (Deg) } \\
\hline & & 21.3 & 11.0 & 5.5 & 2.8 \\
\hline $\begin{array}{l}1 \\
2 \\
3 \\
4 \\
5\end{array}$ & 3.1 & $\begin{array}{l}51 \\
35 \\
43 \\
46 \\
38\end{array}$ & $\begin{array}{l}74 \\
37 \\
48 \\
70 \\
53\end{array}$ & $\begin{array}{l}74 \\
50 \\
53 \\
80 \\
58\end{array}$ & $\begin{array}{l}78 \\
58 \\
60 \\
79 \\
68\end{array}$ \\
\hline $\begin{array}{l}1 \\
2 \\
3 \\
4 \\
5 \\
6\end{array}$ & 3.2 & $\begin{array}{l}46 \\
41 \\
58 \\
37 \\
49 \\
46\end{array}$ & $\begin{array}{l}65 \\
51 \\
59 \\
50 \\
59 \\
55\end{array}$ & $\begin{array}{l}75 \\
61 \\
56 \\
55 \\
58 \\
68\end{array}$ & $\begin{array}{l}80 \\
67 \\
57 \\
59 \\
68 \\
72\end{array}$ \\
\hline & & $\begin{array}{r}44.5 \\
6.7 \\
\end{array}$ & $\begin{array}{l}56.5 \\
10.5 \\
\end{array}$ & $\begin{array}{l}62.5 \\
10.1 \\
\end{array}$ & $\begin{array}{r}67.8 \\
8.6 \\
\end{array}$ \\
\hline
\end{tabular}

estimate the initial distance in meters, gave estimates that varied from 0.1 to $15 \mathrm{~m}$, median $=2.5 \mathrm{~m}$ ). It can then be argued that the individual variation in amount of perceived relative motion was caused by the variation in perceived initial distance.

According to the model (Eq. 1), the initial distance should not influence the perceived relative motion in depth. but this assumption has not yet been empirically tested. and according to the alternative equation. Eq. 2 . there ought to be such an effect. If. by means of Eq. 2 . the perceived infinity is defined as

$$
Z_{\infty}=\operatorname{Lim}_{\Delta x \rightarrow X} Z=K(1-a) / a
$$

then

$$
\left.a=K / K+Z_{\infty}\right)
$$

The perceived infinity can be interpreted as corresponding to the horizon of the perceived space. A plausible assumption is, then, that the perceived infinity does not vary with the perceived initial distance to an object in a given experimental situation and for a given S. Otherwise, the perceived space would shrink and grow with the perceived initial positions of objects in this space. Then, according to Eq. 4, a has to vary with $\mathrm{K}$. More precisely, as $\mathrm{K}$ increases, the perceived relative distance of motion should decrease.

The aim of Experiment 3 was to test this prediction. In order to be able to vary the initial distance systematically, it was necessary to vary the initial size of the transformation patterns. Experiment 3 was then designed to study if initial size and initial distance in covariation had any effect on perceived relative motion in depth.

Experiment 3 consisted of two experiments. Experiments 3.1 and 3.2. The only difference between them was that the produced variation in perceived initial distance was measured in Experiment 3.2 but not in Experiment 3.1 .

\section{Method}

Experimental technique. The same experimental situation was used as in Experiment 1, but the technique of generating the transformation patterns was different. The patterns consisted of outlined squares of different initial sizes generated by means of a digital computer (LINC-8). They were presented one by one. The rate of change was constant $(21 \mathrm{c} / \mathrm{sec})$, and the amount of change was $98.5 \%$.

In order to get a measure of perceived relative motion in depth, the Ss could mark off and adjust any distance in the motion by stopping the square for $0.5 \mathrm{sec}$ at the appropriate moment. This technique had also been used in the earlier experiments (Marmolin, 1973). a technique which made it possible to get a measure of perceived amount of motion in a convenient way.

Subjects and procedure. Five $S$ s participated in Experiment 3.1 and six in Experiment 3.2. The Ss' task was just to mark off a distance (from the starting point of the motion) that was perceived to be equal to the initial distance.

Four initial sizes of the square were chosen $(2.8,5.5,11.0$. and $21.3 \mathrm{deg}$ of visual angle). and the patterns were presented twice in randomized order. At each presentation. the perceived motion was measured three times.

In Experiments 3.1 and 3.2. all Ss reported that the smaller the initial size. the larger the initial distance perceived. In order to get a rough measure of this effect. an attempt was made in Experiment 3.2 to measure the perceived initial distance. However, preexperiments had shown that the same experimental situation could not be used. since the period of time between the presentation of two different initial sizes (about $10 \mathrm{sec}$ ) was too long. In this part of Experiment 3.2. therefore. static squares were generated with the same size as the initial size of the transformation patterns. The static squares were presented pairwise. in all six possible combinations. successively and repeatedly. The first member of the pair was presented for $2 \mathrm{sec}$ : after a 1 -sec paus, the next member was presented for 2 sec: then the first again. and so on, as many times as necessary to make the judgments. The different pairs were presented four times in randomized order. The perceived distance was measured by letting the $S s$ judge the ratio between the distance to the nearest and farthest square, according to a variant of the method of constant sum. The distance to the farthest was given the value of 100. and the Ss' task was to estimate the numerical value corresponding to the distance to the nearest square.

\section{Results and Conclusions}

The results of Experiment 3 are summarized in Tables 3 and 4 . For all Ss except one, the perceived relative motion decreased systematically (amount of change corresponding to the initial distance increased) as the initial size of the square decreased (Table 3). It may also be concluded that the initial distance increased as the initial size decreased. As mentioned above, all Ss reported such an effect. Furthermore, Experiment 3.2 showed that there was a systematic relation between the size of the static squares and the perceived distance to these squares (Table 4 ). That this relationship also holds for the transformation patterns could, of course, be questioned as other kinds of stimuli and another situation were used. It may be that the obtained scale 
values are not applicable, but as the Ss reported that the static squares were perceived at approximately the same distance as the initial distance of the transformation patterns, the general trend in Table 4 ought to be valid.

The results of this experiment will be more closely discussed in connection with Experiment 4. At this stage, it can be concluded only that there are strong and systematic effects of initial distance in combination with initial size on perceived relative motion in depth.

\section{Experiment 4}

Experiment 3 showed that initial size and initial distance in covariation strongly influence the perceived relative motion. The purpose of Experiment 4 was to study the effect of initial size alone, i.e., the effect of absolute amount of change in pattern with the same relative amount of change.

\section{Method}

The same experimental situation and technique of generating and presenting the transformation patterns was used as in Experiment 1. However, in order to generate outlined figures, the high frequencies consisted of peak-clipped sinusoidal waves with a phase difference of $90 \mathrm{deg}$.

Six Ss participated in the experiment. The effect of size was measured by the same matching paradigm as in Experiment 1 . The standards were four patterns of different size $(2.8,5.5,11.0$, and $21.3 \mathrm{deg}$ of visual angle), the same as in Experiment 3, and the variable stimuli, a pattern of 5.5-deg size. The rate of change of the standards was $33 \% / \mathrm{sec}$, and the amount of change, $56 \%$. The standard and variable patterns were presented with the contours close together, and therefore the Ss perceived them at the same initial distance.

PSE- $Z$ was measured by the up-and-down method four times at each presentation of the standards. The standards were presented twice in randomized order, once with the change of the variable pattern smaller than the expected threshold, once with larger change.

\section{Results and Conclusions}

The results are given in Table 5 .

An analysis of variance (mixed model, Ss by Size) revealed no significant effects of size $(p<.05)$. This finding is in agreement with the result of Experiment 1. As in other experiments, effects of $S$ and interaction with $\mathrm{S}$ were not interpreted. However, an inspection of

Table 4

Judged Ratio (Means of Individual Means) Between the Different Static Squares of Experiment 3.2 and Calculated Scale Values

\begin{tabular}{ccccc}
\hline $\begin{array}{c}\text { Size } \\
(\text { Deg })\end{array}$ & $\begin{array}{l}21.3 \\
\text { Deg }\end{array}$ & $\begin{array}{c}11.0 \\
\text { Deg }\end{array}$ & $\begin{array}{c}5.5 \\
\text { Deg }\end{array}$ & $\begin{array}{c}2.8 \\
\text { Deg }\end{array}$ \\
\hline 21.30 & & 0.68 & 0.29 & 0.21 \\
11.00 & & & 0.60 & 0.45 \\
5.50 & & &. & 0.80 \\
2.8 & & & & \\
Scale Value & 1.00 & 1.78 & 3.05 & 4.25 \\
\hline
\end{tabular}

Table 5

PSE-Z (Mean Percent Change) for the Standard Patterns Used in Experiment 4

\begin{tabular}{ccccc}
\hline & \multicolumn{4}{c}{ Maximal Size (Deg) } \\
\cline { 2 - 5 } Ss & 21.3 & 11.0 & 5.5 & 2.8 \\
\hline 1 & 57 & 55 & 55 & 60 \\
2 & 73 & 63 & 61 & 58 \\
3 & 63 & 61 & 55 & 64 \\
4 & 64 & 69 & 66 & 56 \\
5 & 63 & 66 & 59 & 59 \\
6 & 71 & 64 & 56 & 66 \\
Mean & 65.2 & 63.0 & 58.7 & 60.5 \\
SD & 5.8 & 4.8 & 4.3 & 3.8 \\
\hline
\end{tabular}

individual data gave no evidence for any systematic effects of size. Just one S (S 2) had a tendency to be systematically affected. It seems reasonable, then, to conclude that perceived motion in depth is not determined by the absolute amount of change but by the relative amount of change, as assumed in the model.

The outcome of Experiment 3 showed that initial distance and size in covariation influence perceived relative motion in depth. In this experiment, no effects of size alone came out. Disregarding possible interactions between initial distance and size, the results indicate, then, that the effective variable in Experiment 3 was perceived initial distance, not size. It should be noted that the effect of size found for S 2 in Experiment 4 does not contradict this conclusion, as this effect should counteract the effect of initial distance.

Experiments 3 and 4, then, confirm the prediction derived from Eq. 2, that perceived relative motion in depth decreases as the perceived initial distance increases. In addition, this fact indicates that the earlier obtained individual differences in amount of perceived relative motion could have been caused by individual differences in perceived initial distance.

\section{GENERAL DISCUSSION}

The aim of this investigation was to study the effects of rate of change, perceived initial distance, and absolute amount of change on perceived relative motion in depth.

The effects of relative rate of change, absolute rate of change, and type of rate of change function were studied. The only temporal property that gave significant effects was the type of rate of change function. A retarded rate of change seems to produce greater motion in depth than does an accelerated one. However, these effects were very weak. The same weak tendency was also found in the earlier study (Marmolin, 1973) when a constant rate of change was compared with a linear retardation. Thus, it seems reasonable to conclude that temporal properties in a changing proximal pattern of light are of little importance for perceived motion in depth, at least from a theoretical 
point of view. The same conclusion was reached by Jansson and Börjesson (1969) concerning perceived direction of rotation and by Börjesson and Hofsten (1971) concerning depth perception from dots in motion.

It was found that perceived initial distance has a rather strong and systematic influence on perceived relative motion in depth. As the perceived initial distance increases, perceived relative motion decreases. This finding is not consistent with the model proposed by Johansson (1964), but it is consistent with the alternative Eq. 2.

Thus, Eq. 2 seems to cover the result of both this study and the earlier study (Marmolin, 1973). The objective of future research will be to test its applicability further-for example, by a direct test of the derived expression (Eq. 4).

Finally: the experiments in this report showed that absolute amount of change is not a determinant of perceived relative motion in depth. The relevant variable is amount of relative change as assumed in the model. This fact, together with the found independence of temporal properties, demonstrates that the visual system acts rather mechanically in decoding the stimulation. The obtained effect of initial distance is also compatible with a projective analysis of the stimulation (Eq. 2), but shows that the stimulus-percept relationship is far more complex than postulated in the model.

This investigation was based on some results obtained in the earlier study (Marmolin, 1973) concerning the relation between perceived relative distance of motion in depth and amount of proximal change. One of the conclusions from this study was that the stimulus-percept formula (Eq. 1) proposed by Johansson (1964) did not predict perceived relative motion in depth. It is now evident that explanations of this finding cannot be based on temporal properties of the pattern used. Future research has to be directed to other alternatives.

Another finding in the earlier study (Marmolin, 1973) was that there were large interindividual differences in amount of perceived relative motion. This variation may have been caused by interindividual differences in perceived initial distance, as there were such differences and as the present study showed initial distance to be an effective variable. This hypothesis must, therefore, be more directly tested before it can be fully accepted. Research is in progress with this objective.

\section{REFERENCES}

Börjesson, E., \& v. Hofsten. C. Determinants of depth perception in motion pattern. Report No.101. Department of Psychology, University of Lppsala. 1971.

Jansson. G.. \& Börjesson. E. Perceived direction of rotary motion. Perception \& Psychophysics, 1969, 6, 19-26.

Johansson, G. Perception of motion and changing form. Scandinavian Journal of Psychology. 1964. 5, 181-208.

Marmolin, H. Visually perceived motion in depth resulting from proximal changes (I). Perception \& Psychophysics, 1973, 14, 133-142.

Marmolin. H., \& Ulfberg. S. Proximal changes of perceived distance of motion in depth. Report No. 125. Department of Psychology. University of Uppsala, 1972.

(Received for publication July 31, 1972: revision received February 12, 1973.) 